\title{
The association between diabetes mellitus and HRQoL of older people in Shanghai
}

\author{
Shiyin Tian ${ }^{1,2+}$, Rui Wang ${ }^{3 \dagger}$, Mengxing Qian ${ }^{2 \dagger}$, Lijuan Liu' ${ }^{1}$, Zhenyi Shao ${ }^{4}$, Cheng Wu ${ }^{3 *}$ and Jinhai Sun ${ }^{{ }^{*}}$
}

\begin{abstract}
Background: This study aimed to explore the association between diabetes mellitus and health-related quality of life (HRQoL) of older people in Shanghai, China, especially regarding the differences in each aspect of the EQ-5D and how large the score gaps are between older people with and without diabetes.

Methods: A total of 11,103 people of either sex older than 60 years were enrolled from 17 districts of Shanghai. The EQ-5D-3L was used to assess the HRQoL of older people. The Wilcoxon rank sum test and t-test were used to compare the difference in $\mathrm{HRQ}$ L between people with or without diabetes. After univariate regression, multiple linear regression and ordinal logistic regression were conducted to evaluate the influence of diabetes mellitus and other confounding variables on the EQ VAS scores and on the five dimensions of the EQ-5D.
\end{abstract}

Results: Twelve percent of all participants had diabetes mellitus, and the proportion was almost the same between men and women. The EQ VAS scores of people with diabetes mellitus were approximately 3.70 points lower than those of people without diabetes $(95 \% \mathrm{Cl}=-4.40,-2.99, p<0.001)$ after adjusting for confounding variables. People with diabetes mellitus had increased problems with mobility $(\mathrm{OR}=1.57,95 \% \mathrm{Cl}=1.33,1.85)$, self-care $(\mathrm{OR}=1.65,95 \%$ $\mathrm{Cl}=1.35,2.01)$, usual activities $(\mathrm{OR}=1.78,95 \% \mathrm{Cl}=1.51,2.11)$, pain/discomfort $(\mathrm{OR}=1.42,95 \% \mathrm{Cl}=1.24,1.64)$, and anxiety/depression $(\mathrm{OR}=1.33,95 \% \mathrm{Cl}=1.07,1.64)$.

Conclusions: This study showed that diabetes mellitus was associated with the HRQoL of older people and that older people with diabetes had poorer performance in every aspect of EQ-5D measurements.

Keywords: Diabetes mellitus, Quality of life, Older people

\section{Backgrounds}

Many countries have a rapidly ageing population, and the global population of people aged 60 years and older will more than double, from 900 million in 2015 to approximately 2 billion, in 2050 [1]. In China, according to the latest report released by the China Development Research Foundation in June 2020, individuals over 65 will make up $14 \%$ of the total population in approximately

\footnotetext{
*Correspondence: wucheng_wu@126.com; jinhaisunsmmu@163.com

†Tian Shiyin, Wang Rui and Qian Mengxing co-first author.

${ }^{1}$ Department of Health Management, Navy military medical university, Shanghai, China

${ }^{3}$ Department of Health Statistics, Navy Military Medical University,

Shanghai, China

Full list of author information is available at the end of the article
}

2022 and reach $27.9 \%$ in 2050 . With the growing number of older people, a surge in the prevalence and incidence of age-associated diseases is inevitable [2]. In addition, older people often more frequently experience poor functional health and are more vulnerable to diseases [3]; hence, the quality of life of the geriatric population is raising increased concern.

Among the many age-associated diseases, diabetes mellitus is one of the most common chronic diseases among older people. The number of people with diabetes rose from 108 million in 1980 to 422 million in 2014 worldwide [4]. People who have diabetes are at a high risk of many complications, such as low-grade inflammation, cardio-cerebrovascular disease and amputation [5], and these complications are sometimes atypical and can take 
a long time to discover [6], which has more serious negative consequences for the health of geriatric people with diabetes. Additionally, the treatment of diabetes encompasses adherence to strict eating guidelines, regular use of antidiabetic drugs and/or insulin, self-monitoring of blood glucose and other long-term treatments [7], all of which influence patient quality of life.

Among the many methods for assessing quality of life, health-related quality of life (HRQoL) is used worldwide and is an important way to measure the impact of chronic disease on people's quality of life [8]; moreover, HRQoL is often used to study the health status of older people [9]. Thus, in this article, we employed HRQoL to measure the association between diabetes mellitus and the quality of life of older people.

Previous studies have identified several chronic diseases that are associated with HRQoL. A study of the relationship between chronic diseases and HRQoL showed that older adults ( $>65$ years) with dementia/ Alzheimer's disease, stroke/transient ischaemic attack (TIA), osteoarthritis/gout/rheumatoid arthritis (RA), and osteoporosis had a lower HRQoL [10], and another study found that depression, stroke, heart disease and cognitive dysfunction had a significantly adverse impact on HRQoL [11]. In a systematic review in 2018, ten articles studied the relationship between diabetes mellitus and HRQoL, but the study with the highest values was conducted on participants with a younger mean age (57.2 years) [12]. There is little literature about how diabetes mellitus influences HRQoL in the geriatric population, and our study aimed to fill this research gap by focusing on the HRQoL of older people with diabetes mellitus.

\section{Methods}

\section{Study design and data source}

The aim of the study was to explore the association between diabetes mellitus and health-related quality of life (HRQoL) of older people in Shanghai, especially regarding the differences in each aspect of the EQ-5D and how large the score gaps are between older people with and without diabetes. All older people with diabetes mellitus in this study were diagnosed with diabetes by a doctor.

The data used in this study were extracted from the fifth National Household Health Survey of Shanghai, China (NHHS), which was conducted in 2013 [13]. The NHHS is a cross-sectional study organised by the National Health Commission of China every five years, and the data were collected by a face-to-face interview conducted by trained staff. The households were sampled using a multistage stratified cluster sampling procedure. Informed consent forms were provided and signed before the interview.

\section{Participants and measurement}

A total of 11,103 older people of either sex aged over 60 years were enrolled from 17 districts of Shanghai, China.

The EQ-5D-3L was used to assess the HRQoL of older people. The EQ-5D consists of 2 parts: the EQ-5D descriptive system and the EQ visual analogue scale (EQ VAS). The EQ-5D descriptive system comprises five dimensions: mobility, self-care, usual activities, pain/discomfort and anxiety/depression; each dimension has 3 levels: no problems, some problems, and extreme problems. The interviewees were asked to describe their health status for each of the five dimensions with the three levels. The EQ VAS is an analogue scale where the endpoints are labelled "Best imaginable health state", worth 100 points, and "Worst imaginable health state", worth 0 points. Respondents were asked to self-rate their health between 0 and 100 points. The EQ VAS can be used as a quantitative measure of health outcomes that reflects the patient's own judgement of their health [14]. The Chinese version of the EQ-5D questionnaire has been demonstrated to assess quality of life [15], and the reliability of the EQ-5D was good in this study (Cronbach's alpha $=0.841$ ).

Diabetes mellitus was assessed by the interviewer with the question "Have you been diagnosed with diabetes mellitus by a doctor?", and the outcome of the question was a binary variable that indicated whether the subject had diabetes mellitus. All older people with diabetes mellitus in this study were aware of their disease and were diagnosed by a doctor.

According to previous studies, several influencing factors should be considered. Body mass index (BMI) groups had a close relationship with HRQoL, so BMI was considered an influencing factor. Additionally, two types of control variables were collected, one of which was sociodemographic variables including age, sex, registered residence, education, marital status, and income source (the classification are shown in Table 1), and the other was health-related behaviours such as smoking, drinking, physical exercise and health examination participation. Smoking status was divided into three levels: everyday smoking, not everyday smoking, and never smoking. Drinking represented whether the participant had consumed alcohol in the previous 12 months. Physical exercise referred to whether the participant had exercised in the past 6 months, and health examination indicated whether the subject had his/her health checked in the past year.

\section{Statistical analysis}

Descriptive statistics were employed to describe the general characteristics of the 11,103 older people. Categorical data and ranked data are presented as numbers 
Table 1 General characteristics of older people according to sex

\begin{tabular}{|c|c|c|c|}
\hline Characteristics & $\begin{array}{l}\text { Male } \\
\text { N (\%) }\end{array}$ & $\begin{array}{l}\text { Female } \\
\mathrm{N}(\%)\end{array}$ & $\begin{array}{l}\text { Total } \\
\mathrm{N}(\%)\end{array}$ \\
\hline \multicolumn{4}{|l|}{ Age } \\
\hline $60-69$ & $3002(57.2)$ & $3144(53.7)$ & $6146(55.4)$ \\
\hline 70-79 & $1436(27.4)$ & $1588(27.1)$ & $3024(27.2)$ \\
\hline$\geq 80$ & $806(15.4)$ & $1127(19.2)$ & 1933(17.4) \\
\hline \multicolumn{4}{|l|}{ Registered residence } \\
\hline Rural & 977(18.6) & $1212(20.7)$ & 2189(19.7) \\
\hline Urban & $4267(81.4)$ & $4647(79.3)$ & $8914(80.3)$ \\
\hline \multicolumn{4}{|l|}{$\mathrm{BMI}\left(\mathrm{kg} / \mathrm{m}^{2}\right)$} \\
\hline$<18.5$ & $300(5.7)$ & $476(8.1)$ & $776(7.0)$ \\
\hline $18.5-23.9$ & 2950(56.3) & $3277(55.9)$ & $6227(56.1)$ \\
\hline $24-27.9$ & 1707(32.6) & 1674(28.6) & $3381(30.5)$ \\
\hline$\geq 28$ & $287(5.5)$ & $432(7.4)$ & $719(6.5)$ \\
\hline \multicolumn{4}{|l|}{ Education } \\
\hline Illiterate & $407(7.8)$ & 1458(24.9) & 1865(16.8) \\
\hline Primary school & 1353(25.8) & $1639(28.0)$ & 2992(26.9) \\
\hline Junior high school & 1643(31.3) & $1473(25.1)$ & $3116(28.1)$ \\
\hline Senior high school and above & $1841(35.1)$ & $1289(22.0)$ & $3130(28.2)$ \\
\hline \multicolumn{4}{|l|}{ Marital status } \\
\hline Single & $82(1.6)$ & $76(1.3)$ & $158(1.4)$ \\
\hline Widowed & $484(9.2)$ & 1579(26.9) & 2063(18.6) \\
\hline Divorced & $52(1.0)$ & $81(1.4)$ & $133(1.2)$ \\
\hline Married & $4626(88.2)$ & $4123(70.4)$ & $8749(78.8)$ \\
\hline \multicolumn{4}{|l|}{ Income source } \\
\hline Oneself or mate & 4990(95.2) & $5413(92.4)$ & $10,403(93.7)$ \\
\hline Children or grandchildren & $146(2.8)$ & $301(5.1)$ & $447(4.0)$ \\
\hline Social relief & $48(0.9)$ & $76(1.3)$ & $124(1.1)$ \\
\hline Other & $60(1.1)$ & $69(1.2)$ & $129(1.2)$ \\
\hline \multicolumn{4}{|l|}{ Smoking } \\
\hline Never & $3261(62.2)$ & $5741(98.0)$ & $9002(81.1)$ \\
\hline Not everyday & $201(3.8)$ & $64(1.1)$ & $265(2.4)$ \\
\hline Everyday & $1782(34.0)$ & $54(0.9)$ & 1836(16.5) \\
\hline \multicolumn{4}{|l|}{ Drinking } \\
\hline Yes & 1764(33.6) & $154(2.6)$ & 1918(17.3) \\
\hline No & $3480(66.4)$ & $5705(97.4)$ & $9185(82.7)$ \\
\hline \multicolumn{4}{|l|}{ Physical exercise } \\
\hline Yes & 2982(56.9) & $3102(52.9)$ & $6084(54.8)$ \\
\hline No & $2262(43.1)$ & $2757(47.1)$ & $5019(45.2)$ \\
\hline \multicolumn{4}{|l|}{ Health examination } \\
\hline Yes & $3212(61.3)$ & $3497(59.7)$ & $6709(60.4)$ \\
\hline No & 2032(38.7) & $2362(40.3)$ & 4394(39.6) \\
\hline \multicolumn{4}{|l|}{ Diabetes mellitus } \\
\hline Yes & $671(12.8)$ & $745(12.7)$ & $1416(12.8)$ \\
\hline No & $4573(87.2)$ & $5114(87.3)$ & $9687(87.2)$ \\
\hline
\end{tabular}

(percentages). Continuous variables are presented as the mean $\pm \mathrm{SD}$. The proportions of the three levels (no problems, some problems, and extreme problems) reported for the five dimensions of the EQ-5D were also described separately for older people with diabetes mellitus and those without; the Wilcoxon rank sum test was performed to compare the differences in each of the five dimensions of the EQ-5D between the people with or without diabetes, and a t-test was used to compare EQ VAS scores between the two groups.

Univariate analysis was conducted to assess the effects of every characteristic on diabetes. A multiple linear regression model was conducted to evaluate the associations of diabetes mellitus with other confounding variables and EQ VAS scores. Multivariate model 1 was adjusted for age, sex, registered residence, BMI, education, marital status, income source, smoking, drinking, physical exercise and health examination. Model 2 was adjusted for all model 1 factors plus hypertension and other chronic diseases. Ordinal logistic regression was conducted to evaluate the associations of diabetes mellitus with the five dimensions of the EQ-5D and adjusted for all the influencing variables in model 2.

BMI groups, marital status and income source were coded as dummy variables. Less than $5 \%$ of the participants had missing data across all the variables. The missing data were imputed to the dominant category for categorical variables and to the mean for continuous variables.

All analyses were conducted using SPSS software, version 22.0 (IBM Corporation, Chicago, IL, USA) with statistical significance set at a $P$ value $<0.05$, and the figure was generated using GraphPad Prism version 7.00 (GraphPad Software, San Diego, CA, USA).

\section{Results}

\section{Characteristics of the study participants}

The characteristics of all participants are shown in Table 1 and are also presented separately according to sex. A total of $12.8 \%$ of all subjects had diabetes mellitus, and the proportions of males and females were $12.8 \%$ and $12.7 \%$, respectively. Moreover, $55.4 \%$ of all the older people were aged 60 to 69 years old, $27.2 \%$ were aged 70 to 79 , and $17.4 \%$ were aged above 80 years old.

\section{The HRQoL of people with or without diabetes mellitus}

The HRQoL scores of older people with or without diabetes mellitus are presented in Table 2. The proportions of people with no problems across the five dimensionsmobility, self-care, usual activities, pain/discomfort and anxiety/depression-were $86.3 \%, 92.0 \%, 88.1 \%, 80.6 \%$ and $93.2 \%$, respectively, and the mean EQ VAS score was $76.1 \pm 13.7$. The results of the Wilcoxon rank sum test and $\mathrm{t}$-test revealed that there were significant differences in each dimension of the EQ-5D and in EQ VAS scores between the diabetes mellitus group and the group 
Table 2 The results of the EQ-5D according to diabetes groups

\begin{tabular}{|c|c|c|c|}
\hline Dimensions and levels & $\begin{array}{l}\text { Diabetes mellitus } \\
\mathrm{N}(\%) / \text { Mean } \pm \text { SD }\end{array}$ & No diabetes mellitus $\mathrm{N}(\%) /$ Mean \pm SD & $\begin{array}{l}\text { Total } \\
\text { N (\%)/Mean } \pm \text { SD }\end{array}$ \\
\hline \multicolumn{4}{|l|}{ Mobility } \\
\hline no problems & 1139(80.4) & $8441(87.1)$ & $9580(86.3)$ \\
\hline some problems & 235(16.6) & $1112(11.5)$ & $1347(12.1)$ \\
\hline extreme problems & $42(3.0)$ & $134(1.4)$ & $176(1.6)$ \\
\hline $\mathrm{p}$ & $<0.001$ & & \\
\hline \multicolumn{4}{|l|}{ Self-care } \\
\hline no problems & 1248(88.1) & 8968(92.6) & $10,216(92.0)$ \\
\hline some problems & $120(8.5)$ & $543(5.6)$ & $663(6.0)$ \\
\hline extreme problems & $48(3.4)$ & $176(1.8)$ & $224(2.0)$ \\
\hline $\mathrm{p}$ & $<0.001$ & & \\
\hline \multicolumn{4}{|l|}{ Usual activities } \\
\hline no problems & $1161(82.0)$ & 8619(89.0) & 9780(88.1) \\
\hline some problems & 190(13.4) & $821(8.5)$ & $1011(9.1)$ \\
\hline extreme problems & $65(4.6)$ & $247(2.5)$ & $312(2.8)$ \\
\hline $\mathrm{p}$ & $<0.001$ & & \\
\hline \multicolumn{4}{|l|}{ Pain/discomfort } \\
\hline no problems & 1045(73.8) & $7901(81.6)$ & 8946(80.6) \\
\hline some problems & $345(24.4)$ & $1701(17.6)$ & 2046(18.4) \\
\hline extreme problems & $26(1.8)$ & $85(0.9)$ & $111(1.0)$ \\
\hline $\mathrm{p}$ & $<0.001$ & & \\
\hline \multicolumn{4}{|l|}{ Anxiety/depression } \\
\hline no problems & 1293(91.3) & $9060(93.5)$ & $10,353(93.2)$ \\
\hline some problems & 114(8.1) & $595(6.1)$ & $709(6.4)$ \\
\hline extreme problems & $9(0.6)$ & $32(0.3)$ & $41(0.4)$ \\
\hline $\mathrm{p}$ & 0.002 & & \\
\hline EQVAS score & $71.8 \pm 14.8$ & $76.8 \pm 13.4$ & $76.1 \pm 13.7$ \\
\hline $\mathrm{p}$ & $<0.001$ & & \\
\hline
\end{tabular}

without diabetes. In all five dimensions, the proportion of subjects with no problems in the group without diabetes was higher than that in the diabetes mellitus group, and the proportion of extreme problems was lower. EQ VAS scores were also higher in older people without diabetes $(p<0.001)$.

\section{The influence of diabetes mellitus on HRQoL adjusted for other characteristics}

The results revealed the significant influence of diabetes and all the other factors on EQ VAS scores (Table 3, unadjusted model). The results showed that geriatric people who did not have diabetes and were younger were more likely to have higher EQ VAS scores. After adjusting for the influencing factors, diabetes mellitus was found to be significantly associated with EQ VAS scores (Table 3, multivariate model 1). The mean EQ VAS score of people who had diabetes mellitus was approximately 4.61 points lower than that of people who did not have diabetes $(95 \%$ $\mathrm{CI}=-5.33,-3.90, p<0.001)$. The model was significant and explained $11.6 \%$ of the variance $(\mathrm{F}=81.66, \mathrm{p}<0.001$, adjusted $\mathrm{R}^{2}=0.116$ ). After considering hypertension and other chronic diseases (Table 3, multivariate model 2), people who had diabetes mellitus were approximately 3.70 points lower than people who did not have diabetes $(95 \% \mathrm{CI}=-4.40,-2.99, p<0.001)$. The model was significant and explained $17.3 \%$ of the variance $(\mathrm{F}=117.46$, $\mathrm{p}<0.001$, adjusted $\mathrm{R}^{2}=0.173$ ).

People with diabetes mellitus were more likely to have problems across the five dimensions of the EQ-5D. They had increased problems with mobility $(\mathrm{OR}=1.57,95 \% \mathrm{CI}=1.33,1.85)$, self-care $(\mathrm{OR}=1.65$, $95 \% \mathrm{CI}=1.35,2.01)$, usual activities $(\mathrm{OR}=1.78,95 \%$ $\mathrm{CI}=1.51,2.11), \quad$ pain/discomfort $\quad(\mathrm{OR}=1.42, \quad 95 \%$ $\mathrm{CI}=1.24,1.64)$, and anxiety/depression $(\mathrm{OR}=1.33$, 95\% CI $=1.07,1.64$ ) (Fig. 1).

\section{Discussion}

The results of the analysis demonstrated that there were significant differences in the five dimensions (mobility, self-care, usual activities, pain/discomfort and anxiety/depression) of the EQ-5D and in EQ VAS 
Table 3 Association of EQ VAS scores with diabetes and other factors

\begin{tabular}{|c|c|c|c|c|c|c|}
\hline \multirow[t]{2}{*}{ Variables } & \multicolumn{2}{|l|}{ Unadjusted model } & \multicolumn{2}{|l|}{ Multivariate model $1^{a}$} & \multicolumn{2}{|l|}{ Multivariate Model $2^{b}$} \\
\hline & $\mathrm{B}(95 \% \mathrm{Cl})$ & $\mathrm{p}$ & $\mathrm{B}(95 \% \mathrm{Cl})$ & $\mathbf{p}$ & $\mathrm{B}(95 \% \mathrm{Cl})$ & $p$ \\
\hline Diabetes & $-5.00(-5.76,-4.25)$ & $<0.001$ & $-4.61(-5.33,-3.90)$ & $<0.001$ & $-3.70(-4.40,-2.99)$ & $<0.001$ \\
\hline Age & $-4.99(-5.31,-4.67)$ & $<0.001$ & & & & \\
\hline Sex & $-1.84(-2.35,-1.34)$ & $<0.001$ & & & & \\
\hline Registered residence & $-0.93(-1.57,-0.29)$ & 0.004 & & & & \\
\hline \multicolumn{7}{|l|}{$\mathrm{BMI}\left(\mathrm{kg} / \mathrm{m}^{2}\right)$} \\
\hline$<18.5$ vs. $\geq 28$ & $-3.47(-4.85,-2.09)$ & $<0.001$ & & & & \\
\hline $18.5-23.9$ vs. $\geq 28$ & $0.93(-0.12,1.98)$ & 0.081 & & & & \\
\hline $24.0-27.9$ vs. $\geq 28$ & $2.34(1.25,3.44)$ & $<0.001$ & & & & \\
\hline Education & $1.10(0.86,1.34)$ & $<0.001$ & & & & \\
\hline \multicolumn{7}{|l|}{ Marital status } \\
\hline Single vs. Married & $-3.17(-5.31,-1.02)$ & 0.004 & & & & \\
\hline Widowed vs. Married & $-5.05(-5.70,-4.40)$ & $<0.001$ & & & & \\
\hline Divorced vs. Married & $0.43(-1.90,2.77)$ & 0.716 & & & & \\
\hline \multicolumn{7}{|l|}{ Income source } \\
\hline Oneself or mate vs. Other & $5.60(4.56,6.64)$ & $<0.001$ & & & & \\
\hline Children or grandchildren vs. Other & $-5.96(-7.25,-4.67)$ & $<0.001$ & & & & \\
\hline Social relief vs. Other & $-6.67(-9.09,-4.26)$ & $<0.001$ & & & & \\
\hline Smoking & $1.63(1.29,1.97)$ & $<0.001$ & & & & \\
\hline Drinking & $3.65(2.98,4.32)$ & $<0.001$ & & & & \\
\hline Physical exercise & $3.57(3.07,4.08)$ & $<0.001$ & & & & \\
\hline Health examination & $1.64(1.12,2.16)$ & $<0.001$ & & & & \\
\hline
\end{tabular}

${ }^{a}$ Model 1: Control variables including age, sex, registered residence, BMI, education, marital status, income source, smoking, drinking, physical exercise and health examination were entered in the model

${ }^{\mathrm{b}}$ Model 2: Model 2 is multivariate model 1 plus hypertension and other chronic diseases

\begin{tabular}{|l|ccccc|}
\hline \multicolumn{1}{|c|}{ Five Dimensions } & OR & lower & upper & pvalue \\
\hline
\end{tabular}

scores between older people with or without diabetes mellitus. The EQ VAS scores of people who had diabetes mellitus were approximately 3.70 points lower than those of people who did not have diabetes (95\% $\mathrm{CI}=-4.40,-2.99, p<0.001)$ after adjusting for confounding variables. The older people with diabetes 
mellitus in this study indicated that they were aware of their disease and had been diagnosed by a doctor.

The strength of the study is that it is a randomised survey of a large sample, and the results are reliable and can reveal the association between diabetes mellitus and HRQoL. The limitation of the study is whether diabetes mellitus was reported by the older participants, which means that some diabetes patients were unlikely to know they had diabetes and had not been diagnosed by a doctor, which led to a lower incidence rate of diabetes in the study. However, there was no measurement of glucose values or haemoglobin $\mathrm{A} 1 \mathrm{C}$ in the original design. Additionally, because the survey database removed private information, we could not sample some of the subjects to measure their glucose values or haemoglobin A1C to estimate the extent of undiagnosed diabetes. This limitation may lead to underestimation of the prevalence of diabetes in older people.

Previous studies on the associations between diabetes and some aspects of quality of life in older people showed relevant findings, and the current study provides more updated results. People with diabetes were more likely to have problems with mobility because of the complications of chronic disease, which even led to disability [16]. Similar findings were reported in a study that carefully measured physical mobility, including running, lifting weight, or walking approximately $100 \mathrm{~m}$ [17]. A previous studies concluded that diabetes patients experience limitations in mobility tasks, general physical activities, and leisure and social activities [18], but few studies have investigated the relationship between diabetes mellitus and self-care or usual activities. Pain or discomfort is more common in people with diabetes, but there was a sex difference not detected in our study. Women with diabetes experienced more frequent and intense pain than women without diabetes, and pain was often felt in the shoulders, knees, and upper extremities; however, the corresponding difference was not observed in men [19]. The injection of insulin also caused mild discomfort in diabetes patients [20]. A meta-analysis showed that people with diabetes often have back pain, but a direct causal link was not established [21]. There are also studies about the association between diabetes and anxiety or depression. A meta-analysis indicated an association between anxiety and diabetes [22], and depression often co-occurs with diabetes [23]. There was also a sex difference in that the effects of mental health symptoms appeared to be stronger in men with diabetes than in women [24]. The EQ VAS scores of older people with diabetes mellitus were lower than those of people without diabetes, which means that the geriatric population with diabetes had a lower HRQoL. A study on diabetes patients who were 18 years and older showed a negative effect of diabetes on
EQ VAS scores [25], and a similar conclusion was made in a Vietnamese study, indicating that older diabetic outpatients had lower EQ VAS scores $(57.5 \pm 14.4)$ [26]. In our study, the mean EQ VAS score of older people with diabetes was $71.8 \pm 14.8$, which was much higher than that in the Vietnamese study. One possible reason is that in the Vietnamese study, the participants were outpatients who may suffer more from the disease, and our study was community-based. Another possible reason is that Shanghai has a more advanced economy and medical care than Vietnam, and $80.3 \%$ of the interviewees were urban residents, so older people with diabetes in Shanghai have a better HRQoL than those in Vietnam.

The present study demonstrated that diabetes mellitus does have a negative impact on the HRQoL of older people in Shanghai across all five dimensions of the EQ-5D. Emerging evidence highlights the adverse impact of diabetes mellitus on the HRQoL of older people, and the management of diabetes mellitus seems to be important for improving the HRQoL of the geriatric population. There was promising improvement in the management of diabetes mellitus, such as proper care, adherence to diabetes guidelines and screening for diabetes-related complications [27]. Older people with diabetes may achieve a better HRQoL by controlling glycaemia and preventing late-term complications, and the government can promote a heathier geriatric population by improving health care system performance.

There could be more influencing factors that were not considered and included in the analysis due to the original survey design, which should be taken into account in further research. Moreover, the glucose values or haemoglobin A1C could be measured to include the currently undiagnosed diabetic people to the diabetes population to obtain a more accurate estimate of how large the gap in EQ VAS scores is between diabetic people and nondiabetic people.

\section{Conclusions}

This study showed that diabetes mellitus was associate with the HRQoL of older people in Shanghai and that older people with diabetes had poorer performance in every aspect of EQ-5D measurements. They were more vulnerable to problems with mobility, self-care, usual activities, pain/ discomfort and anxiety/depression and had lower EQ VAS scores. The older population with diabetes mellitus should be given more attention by individuals and medical providers to achieve a better HRQoL.

\section{Abbreviations}

HRQoL: Health-related quality of life; EQ-5D: European Quality of Life-5 Dimensions; EQ VAS: European Quality of Life Visual Analogue Scale; Cl: Confidence interval; OR: Odds ratio; TIA: Transient ischaemic attack; RA: Rheumatoid arthritis; NHHS: National Household Health Survey; BMI: Body mass index; SD: Standard deviation. 


\section{Acknowledgements}

We would like to acknowledge the Centre for Health Statistics and Information of Shanghai for their cooperation. We would also like to thank all study participants for their time to be interviewed.

\section{Authors' contributions}

TS was a major contributor to the design of the study and wrote the original draft. WR and LL reviewed and edited the manuscript. QM performed data curation and collaborated in the design of the study. SZ collaborated in the interpretation of data. WC collaborated in data analysis and reviewed the manuscript. SJ supervised the study and reviewed the manuscript. All authors read and approved the final manuscript.

\section{Funding}

The research was funded by the project of the "Three-Year Action Plan for Strengthening Public Health System in Shanghai (2020-2022) Subject Chief Scientist" (GWV10.2-XD05) and "Natural Science Foundation of Shanghai" (19ZR1469800).

\section{Availability of data and materials}

The data that support the findings of this study are available from the Centre for Health Statistics and Information of Shanghai, but restrictions apply to the availability of these data, which were used under licence for the current study and so are not publicly available. Data are, however, available from the authors upon reasonable request and with permission from the Centre for Health Statistics and Information of Shanghai.

\section{Declarations}

\section{Ethics approval and consent to participate}

All methods were carried out in accordance with the Declaration of Helsinki. The survey was approved by the ethics committee of the Ministry of Health of Shanghai. All respondents were read a statement that explained the purpose of the survey and gave consent to continue, and informed consent was obtained from all the participants in the current study.

\section{Consent for publication}

Not applicable.

\section{Competing interests}

The authors declare that they have no competing interests.

\section{Author details}

${ }^{1}$ Department of Health Management, Navy military medical university, Shanghai, China. ${ }^{2}$ Medical Department, Shanghai Changzheng Hospital, Shanghai, China. ${ }^{3}$ Department of Health Statistics, Navy Military Medical University, Shanghai, China. ${ }^{4}$ Centre for Health Statistics and Information of Shanghai, Shanghai, China.

Received: 31 July 2021 Accepted: 26 October 2021 Published online: 03 November 2021

\section{References}

1. WHO.Elder Abuse. Available from: https://www.who.int/news-room/factsheets/detail/elder-abuse. Accessed 1 Dec 2020.

2. Fang EF, Scheibye-Knudsen M, Jahn HJ, Li J, Ling L, Guo H, et al. A research agenda for aging in China in the 21 st century. Ageing Res Rev. 2015;24:197-205.

3. Etxeberria I, Urdaneta E, Galdona N. Factors associated with healthrelated quality of life (HRQoL): differential patterns depending on age. Qual Life Res. 2019;28(8):2221-31.

4. WHO.Diabetes. Available from: https://www.who.int/news-room/factsheets/detail/diabetes. Accessed 1 Dec 2020

5. Harding JL, Pavkov ME, Magliano DJ, Shaw JE, Gregg EW. Global trends in diabetes complications: a review of current evidence. Diabetologia. 2019;62(1):3-16.
6. Schlienger JL. Complications du diabète de type 2. Press Medicale. 2013:42(5):839-48.

7. Da Silva JA, De Souza ECF, Echazú Böschemeier AG, Da Costa CCM, Bezerra HS, Feitosa EELC. Diagnosis of diabetes mellitus and living with a chronic condition: participatory study. BMC Public Health. 2018:18(1):1-8.

8. GHG, DHF, DLP. Measuring health-related quality of life. Ann Intern Med. 1993:118(8):622-9.

9. Kim Y, Lee E. The association between elderly people's sedentary behaviors and their health-related quality of life: focusing on comparing the young-old and the old-old. Health Qual Life Outcomes. 2019;17(1):1-9.

10. Ge L, Ong R, Yap CW, Heng BH. Effects of chronic diseases on healthrelated quality of life and self-rated health among three adult age groups. Nurs Health Sci. 2019:21(2):214-22.

11. Pan CW, Cong XL, Zhou HJ, Wang XZ, Sun HP, Xu Y, et al. Evaluating health-related quality of life impact of chronic conditions among older adults from a rural town in Suzhou, China. Arch Gerontol Geriatr. 2018;76(June 2017):6-11.

12. Zhou T, Guan H, Yao J, Xiong X, Ma A. The quality of life in Chinese population with chronic non-communicable diseases according to EQ-5D-3L: a systematic review. Qual Life Res. 2018;27(11):2799-814.

13. The Fifth National Health Service survey in Shanghai. Available from: http:// wsjkw.sh.gov.cn/zhgl2/20180815/0012-57676.html. Accessed 1 Dec 2020.

14. EQ-5D-3L. Available from: https://euroqol.org/eq-5d-instruments/eq-5d31-about/. Accessed 1 Dec 2020

15. Luo N, Chew LH, Fong KY, Koh DR, Ng SC, Yoon KH, et al. Do English and Chinese EQ-5D versions demonstrate measurement equivalence? An exploratory study. Health Qual Life Outcomes. 2003;1:1-7.

16. Fishman El. Incident diabetes and mobility limitations: reducing bias through risk-set matching. J Gerontol Ser A Biol Sci Med Sci. 2014;70(7):860-5

17. Nascimento CDM, Mambrini JVDM, De Oliveira CM, Giacomin KC, Peixoto SV. Diabetes, hypertension and mobility among Brazilian older adults: findings from the Brazilian National Household Sample Survey (1998, 2003 and 2008). BMC Public Health. 2015;15(1):1-7.

18. Golden SH Jr. RJT. Diabetes, race, and functional limitations in older U.S. men and women. Diabetes Res Clin Pr. 2015;108(3):390-7.

19. Karjalainen M, Saltevo J, Tiihonen M, Haanpää M, Kautiainen H, Mäntyselkä P. Frequent pain in older people with and without diabetes - Finnish community based study. BMC Geriatr. 2018;18(1):1-8.

20. Karges B, Muche R, Moritz M, Riegger I, Debatin KM, Heinze E, et al. Low discomfort and pain associated with intensified insulin therapy in children and adolescents. Diabetes Res Clin Pract. 2008;80(1):96-101.

21. Pozzobon D, Ferreira ML. Is there an association between diabetes and neck and back pain? - a systematic review. Osteoarthr Cartil. 2018;26:S426.

22. Smith KJ, Deschênes SS, Schmitz N. Investigating the longitudinal association between diabetes and anxiety: a systematic review and meta-analysis. Diabet Med. 2018;35(6):677-93.

23. Park M, Reynolds CF. Depression among older adults with diabetes mellitus. Clin Geriatr Med. 2015;31(1):117-37.

24. Naicker K, Johnson JA, Skogen JC, Manuel D, Øverland S, Sivertsen B, et al. Type 2 diabetes and comorbid symptoms of depression and anxiety: Iongitudinal associations with mortality risk. Diabetes Care. 2017;40(3):352-8.

25. Parik PC, Patel VJ. Health-related quality of life of patients with type 2 diabetes mellitus at a tertiary Care Hospital in India Using EQ 5D 5L. Indian J Endocrinol Metab. 2019;23(4):407-11.

26. Nguyen HTT, Moir MPI, Nguyen TX, Vu AP, Luong LH, Nguyen TN, et al. Health-related quality of life in elderly diabetic outpatients in Vietnam. Patient Prefer Adherence. 2018;12:1347-54.

27. Rahman MS, Akter S, Abe SK, Islam MR, Mondal MNI, Rahman JAMS, et al. Awareness, treatment, and control of diabetes in Bangladesh: a nationwide population-based study. PLoS One. 2015;10(2):1-14.

\section{Publisher's Note}

Springer Nature remains neutral with regard to jurisdictional claims in published maps and institutional affiliations. 